\title{
Distinct esophageal adenocarcinoma molecular subtype has subtype-specific gene expression and mutation patterns
}

Xiangqian Guo ${ }^{1,2^{*}} \mathbb{D}$, Yitai Tang ${ }^{3}$ and Wan Zhu ${ }^{4}$

\begin{abstract}
Background: Esophageal carcinoma (EC), consists of two histological types, esophageal squamous carcinoma (ESCC) and esophageal adenocarcinoma (EAC). EAC accounted for $10 \%$ of EC for centuries; however, the prevalence of EAC has alarmingly risen 6 times and increased to about $50 \%$ of EC in recent 30 years in the western countries, while treatment options for EAC patients are still limited. Stratification of molecular subtypes by gene expression profiling methods had offered opportunities for targeted therapies. However, the molecular subtype in EAC has not been defined. Hence, Identification of EAC molecular subtypes is needed and will provide important insights for future new therapies.

Results: We performed meta-analysis of gene expression profiling data on three independent EAC cohorts and showed that there are two common molecular subtypes in EAC. Each of the two EAC molecular subtypes has subtype specific expression patterns and mutation signatures. Genes which were over-expressed in subtype I EACs rather than subtype II EAC cases, were enriched in biological processes including epithelial cell differentiation, keratinocyte differentiation, and KEGG pathways including basal cell carcinoma. TP53 and CDKN2A are significantly mutated in both EAC subtypes. 24 genes including SMAD4 were found to be only significantly mutated in subtype I EAC cases, while 30 genes including ARIDIA are only significantly mutated in subtype || EACs.
\end{abstract}

Conclusion: Two EAC molecular subtypes were defined and validated. This finding may offer new opportunities for targeted therapies.

Keywords: Esophageal adenocarcinoma, Molecular subtype, Gene expression, Mutation, Therapy

\section{Background}

Esophageal carcinoma (EC) is the sixth most common cause of cancer death in the world [1]. Based on the 2017 estimates by the American Cancer Society, approximate 16,940 new EC cases (13,360 in men and 3,580 in women) were diagnosed and about 15,690 deaths (12,720 in men and 2,970 in women) were estimated. EC consists of two histological types, esophageal squamous carcinoma (ESCC) and esophageal adenocarcinoma (EAC). Current treatments for both types of EC

\footnotetext{
* Correspondence: xqguo@henu.edu.cn

${ }^{1}$ Department of Preventive Medicine, Joint National Laboratory for Antibody Drug Engineering, Institute of Biomedical Informatics, School of Basic Medical Sciences, Henan University, Kaifeng 475004, China

${ }^{2}$ Cell Signal Transduction Laboratory, Henan University, Kaifeng 475004, China

Full list of author information is available at the end of the article
}

are similar, including chemotherapy, radiation therapy and surgery whilst surgery is the most common treatment method. Despite the static low proportion (approximate 10\%) of EAC in EC in the Asian countries, there is an alarming increase of EAC in western countries in recent 30 years, which makes EAC account for half of all the EC cases and as one of the fastest growing malignancies in the US. Though the survival rate of ESCC patients has been improved in recent years, the death rate is still high for EAC patients, suggesting that more efforts should be placed on studying EAC.

In the past decade, the success in molecular stratification and identification of a number types of tumors, e.g. breast cancer, lung cancer, bladder cancer, colon cancer and leiomyosarcoma, into distinct subtypes lead to significant improvement of our knowledge in these malignancies. More importantly, these new findings have led 
to discoveries of novel targeted therapeutic approaches for treating these cancers [2-19]. However, little is known about the molecular heterogeneity (subtypes) of EAC and no targeted therapy currently exists for EAC. Therefore, recognition of different molecular subtypes for EAC will not only improve our understanding of the mechanisms underlining tumorigenesis and tumor progress; but the successful identification of EAC molecular subtypes and the diagnostic markers for these subtypes will also lay the foundation for the development of targeted therapies for EAC.

Within this study, we performed meta-expressionprofiling analysis in three independent big cohorts of EAC, and demonstrated that there are two distinct molecular subtypes of EAC. Furthermore, each of these subtypes has distinct expression pattern and mutation profile.

\section{Result}

Consensus clustering identified two reproducible molecular subtypes of esophageal adenocarcinoma

Gene expression profiles of 88, 75 and 52 cases of EAC were collected from three independent cohorts, including TCGA [20], GSE13898 [21] and GSE19417 [22]. Clinicopathological characteristics of each cohort were described in Additional file 1: Table S1.

In order to determine the number of subtypes of EAC, consensus clustering was performed on each of the three datasets independently after filtered with standard deviation. Despite distinct profiling methods used by these three independent datasets, we consistently found two robust and stable subtypes in all three datasets (Fig. 1). The ratio of subtype I to subtype II EAC patients was 1:1.5, 1:1.7 and 1:0.7 for dataset TCGA, GSE13898 and GSE19417, respectively (Additional file 1: Table S1). Further analysis using SigClust demonstrated that the two subtypes in each cohort were all statistically significantly different (Fig. 1c). Subsequent Silhouette analysis indicated that $68 \%(60 / 88), 99 \%(74 / 75)$ and $100 \%(52 / 52)$ of samples in TCGA, GSE13898 and GSE19417 had positive silhouette values validating the assignments from consensus clustering. The samples with positive silhouette values were defined as "core samples" for subsequent analysis as performed previously [12, 23], unless otherwise stated (Fig. 1d).

To analyze the reproducibility of molecular subtypes between independent cohorts, subclass mapping was performed. Subclass mapping analysis on EAC cases with positive silhouette values showed that subtype I EAC were significantly reproducible among all the cohorts, while subtype II EAC were significantly reproducible in two of the three cohorts (TCGA and GSE13898) (Fig. 1e). The possible reasons for the inconsistent reproducibility of subtype II EAC in GSE19417 cohort might be the disproportionate EAC population in GSE19417 (EAC ratio of two subtypes 1:0.7 in GSE19417 vs.1:1.5/ 1:1.7 in other two cohorts) and different gene expression profiling methods used.

\section{Clinical features of esophageal adenocarcinoma molecular subtypes in three datasets}

The T-staging (size or direct extent of the primary tumor) was found to be significantly different between two EAC molecular subtypes in GSE13898 $(p=0.0009)$. There is no association between molecular subtypes with differentiation $(p=0.9488)$, grade $(p=0.3249$ in TCGA, $p=0.5345$ in GSE13898), alcohol $(p=0.8195)$, smoke $(p=0.5569)$, positive nodes $(p=0.2886)$, or origin of countries $(p=$ 0.1326) (Additional file 1: Table S1). In addition, there is no significant difference of overall survivals between the two EAC molecular subtypes in each of the three datasets. These association analyses indicate that the EAC molecular subtypes are relatively independent of current clinical features.

\section{Identification of biological pathways and processes representative of two molecular subtypes}

To identify the differentially expressed genes between two molecular subtypes of EAC, SAMseq analysis on EAC cases with positive silhouette values was performed between subtype I and subtype II EAC using the TCGA dataset. A total of 678 genes were significantly over-expressed in subtype I cases than subtype II cases including gene NGFR, CXCR2 and ATP6VOA4, while no genes were identified to be over-expressed in subtype II than in subtype I cases by SAMseq analysis (Additional file 1: Table S2). These 678 genes were enriched in biological processes including epithelial cell differentiation, keratinocyte differentiation (Additional file 1: Table S3), and were enriched in KEGG pathways including basal cell carcinoma (Additional file 1: Table S4).

In addition, Gene Set Enrichment Analysis (GSEA) was performed on EAC cases with positive silhouette values to identify subtype specific biological processes, signaling pathways and potential biomarkers. Subtype I EAC was enriched by curated gene sets including BASAL set (HUPER_BREAST_BASAL_VS_LUMINAL_ UP) and WANG set (WANG_BARRETTS_ESOPHAGUS_AND_ESOPHAGUS_CANCER_DN). BASAL set consisted of genes that were up-regulated in basal mammary epithelial cells compared to the luminal ones [24]. WANG set was comprised of genes that were down-regulated in EAC and Barret's esophagus (BE) relative to normal esophagi (Fig. 2) [25]. The unsupervised hierarchical clustering of normal esophageal tissues, subtype I, subtype II and BE, showed that subtype II shared the most similar expression patterns with $\mathrm{BE}$ with the notion that $\mathrm{BE}$ was reported to be the precursor of classic EAC while 
A

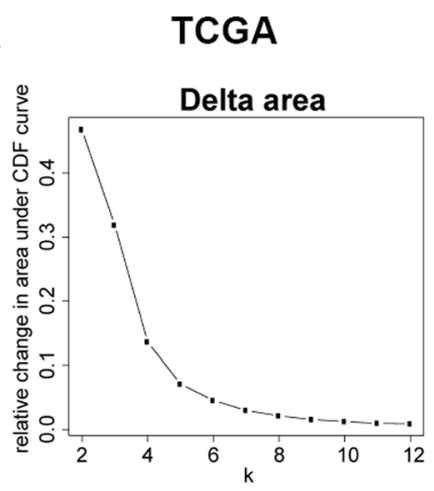

B

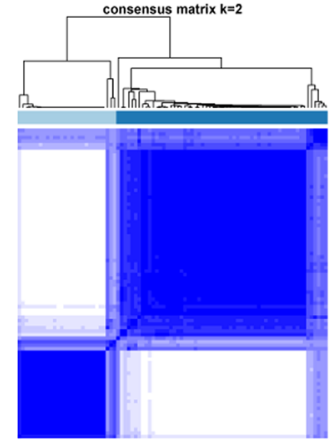

\begin{tabular}{|l|}
\hline$ㅁ$ \\
\hline
\end{tabular}
C SigClust:

$p=0.007$

D Silhouette Plot for $\mathrm{k}=\mathbf{2}$

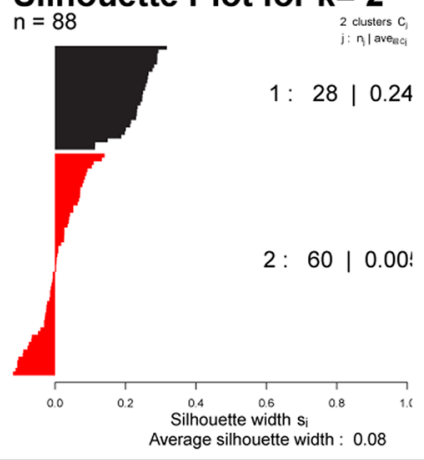

E

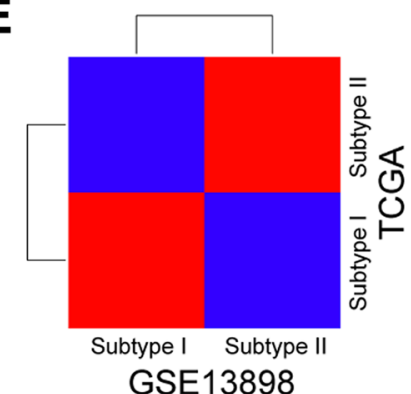

GSE13898
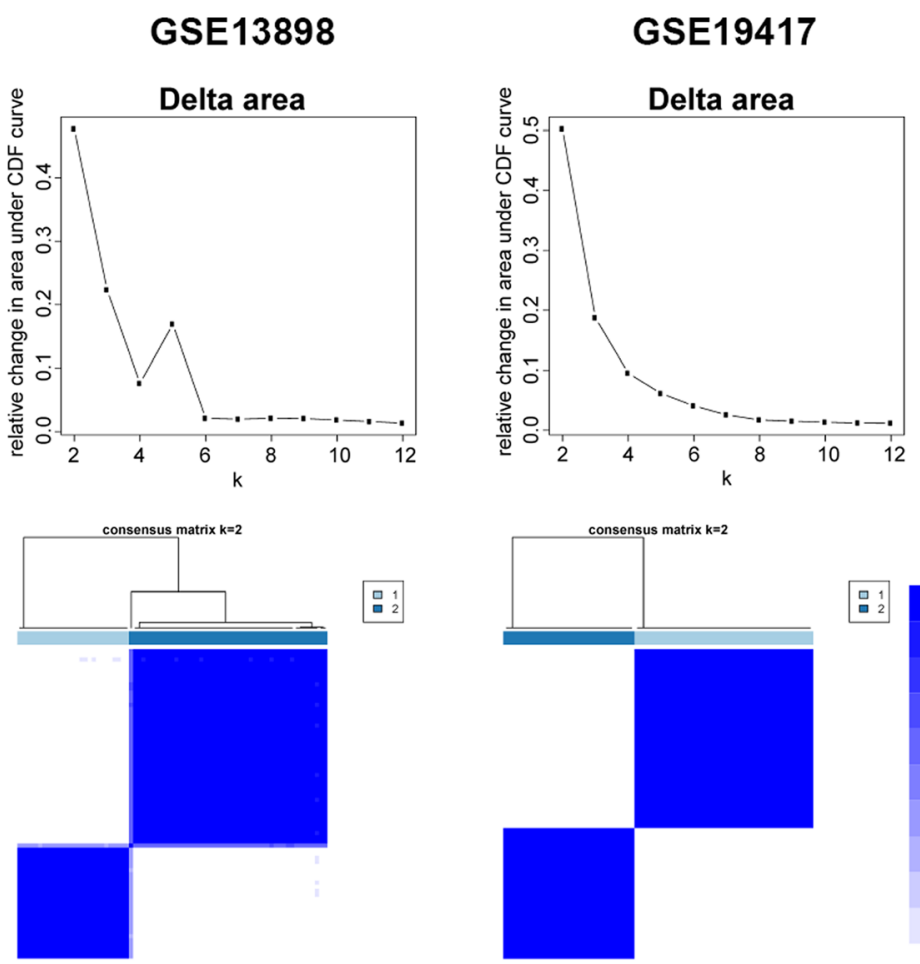

\begin{tabular}{|l|l|}
\hline$a_{1}$ & 1 \\
\hline
\end{tabular}

SigClust:

$$
p=0
$$

\section{Silhouette Plot for $\mathrm{k}=\mathbf{2}$}
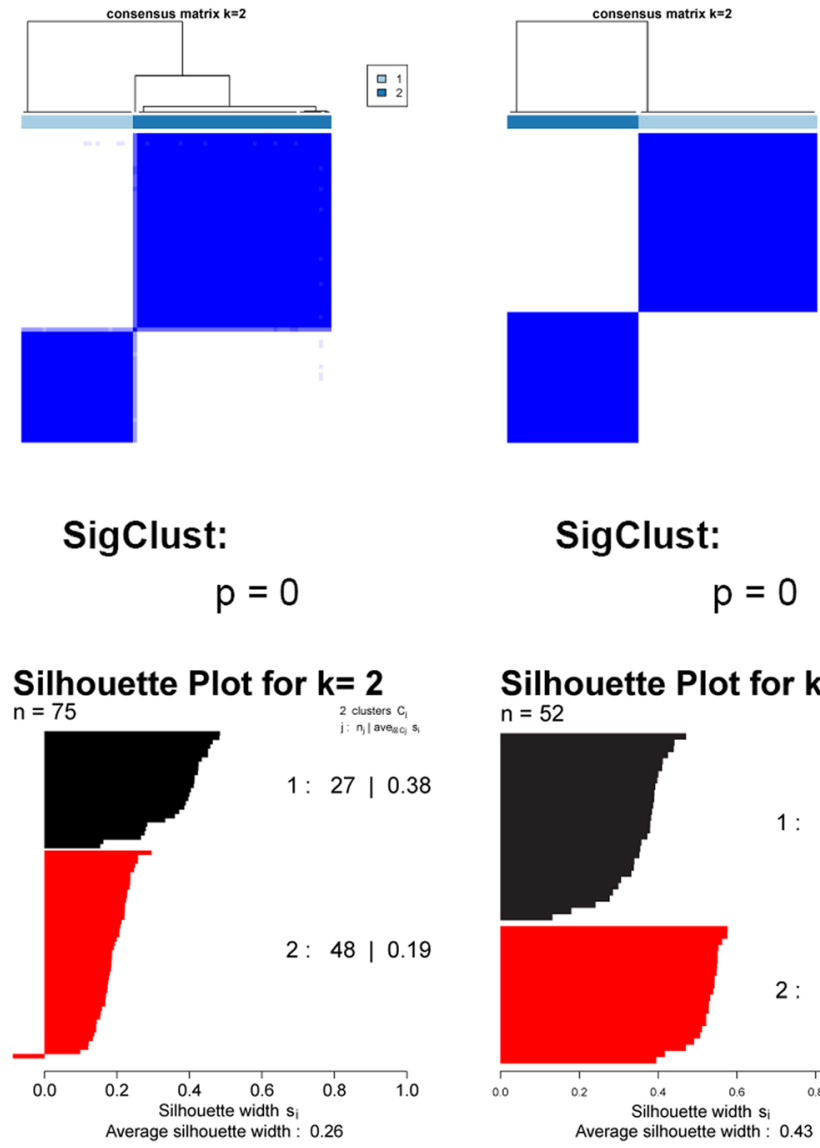

\begin{tabular}{ll}
\hline 뭄 1 \\
2
\end{tabular}
SigClust:

$p=0$

Silhouette Plot for $\mathbf{k}=\mathbf{2}$

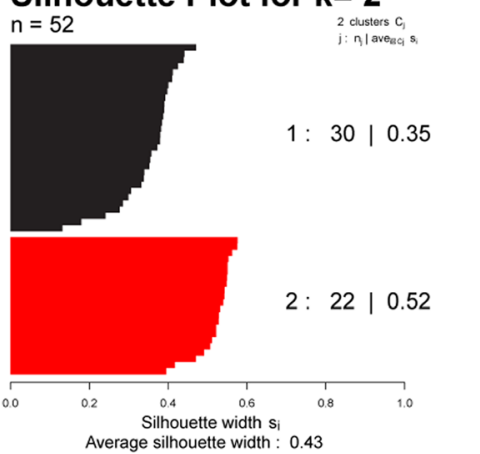

Fig. 1 (See legend on next page.)
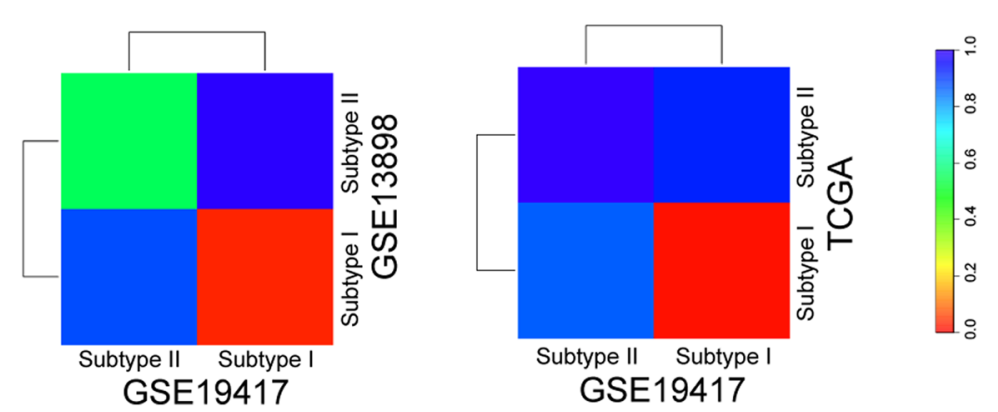
(See figure on previous page.)

Fig. 1 Identification of two molecular subtypes of esophageal adenocarcinoma. a Area under empirical cumulative distribution plots $(k=2$ to $k=12)$ determined the two optimal molecular subtypes of EAC. ( $\mathrm{k}$ denotes the number of clusters). $\mathbf{b}$ Consensus matrix displaying the two robust subgroups of EAC were defined by gene expression. c Significance analysis of subtype classifications in each dataset was determined by SigClust. $\mathbf{d}$ Silhouette analysis of EAC samples based on the assignments from Consensus Clustering. e Subclass association (SA) matrix of molecular subtypes between three cohorts. The rainbow bar indicates the FDR-corrected $p$ value

subtype I EAC cases were closest to normal tissues (Fig. 3). When unsupervised hierarchical clustering the cases of two subtypes of EAC, squamous esophageal carcinoma and gastric carcinoma, we found that subtype I EAC cases shared most similar molecular expression profiles with gastric carcinoma while subtype II EAC cases overwhelmingly co-clustered with squamous carcinoma (Fig. 4). In addition, the genes shared by subtype I EAC and gastric cancer were enriched in KEGG pathways including drug metabolism-other enzymes, while KEGG pathways, including Drug metabolism-cytochrome P450 were common for subtype II EAC and squamous carcinoma (Fig. 4 and Additional file 1: Table S4).

The comprehensive study of esophageal carcinoma from TCGA [26] revealed that EAC strongly resembled CIN (Chromosomal Instability) gastric adenocarcinoma. Our current study further divided EAC population into sub-populations, subtype I and subtype II EACs, and showed that subtype I EAC shared more common expression patterns with gastric adenocarcinoma (Fig. 4).
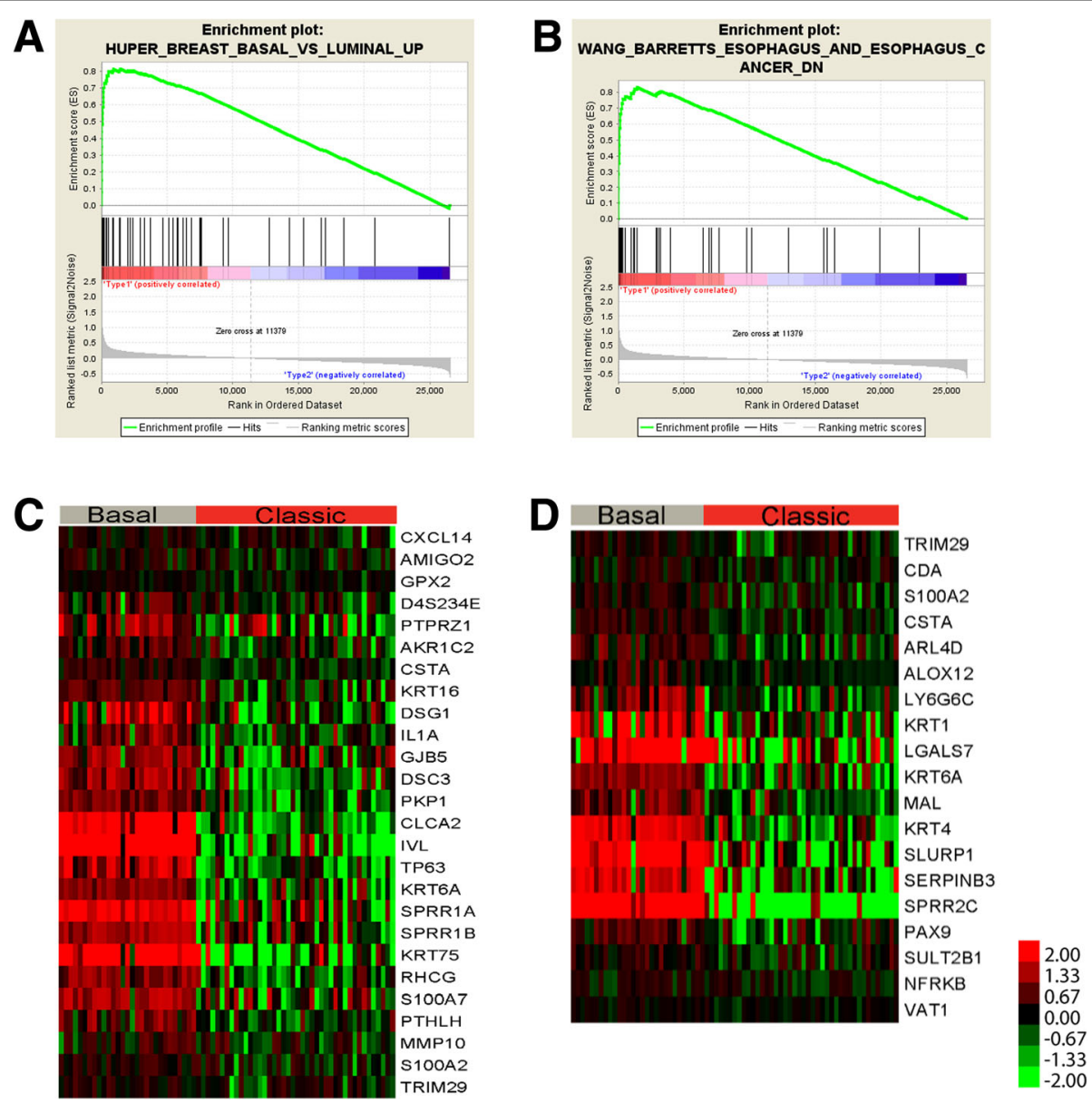

Fig. 2 GSEA gene sets and relative expression heatmap. a. GSEA Enrichment plot for gene set HUPER_BREAST_BASAL_VS_ LUMINAL_UP. b GSEA Enrichment plot for gene set WANG_BARRETTS_ESOPHAGUS_AND_ESOPHAGUS_CANCER_DN. c Heatmap of core enrichment genes for gene set HUPER_BREAST_BASAL_VS_LUMINAL_UP. d Heatmap of core enrichment genes for gene set WANG_BARRETTS_ESOPHAGUS_AND_ESOPHAGUS_CANCER_DN. Note. "Basal" and "Classic" stand for Subtype I and Subtype II EAC, respectively 


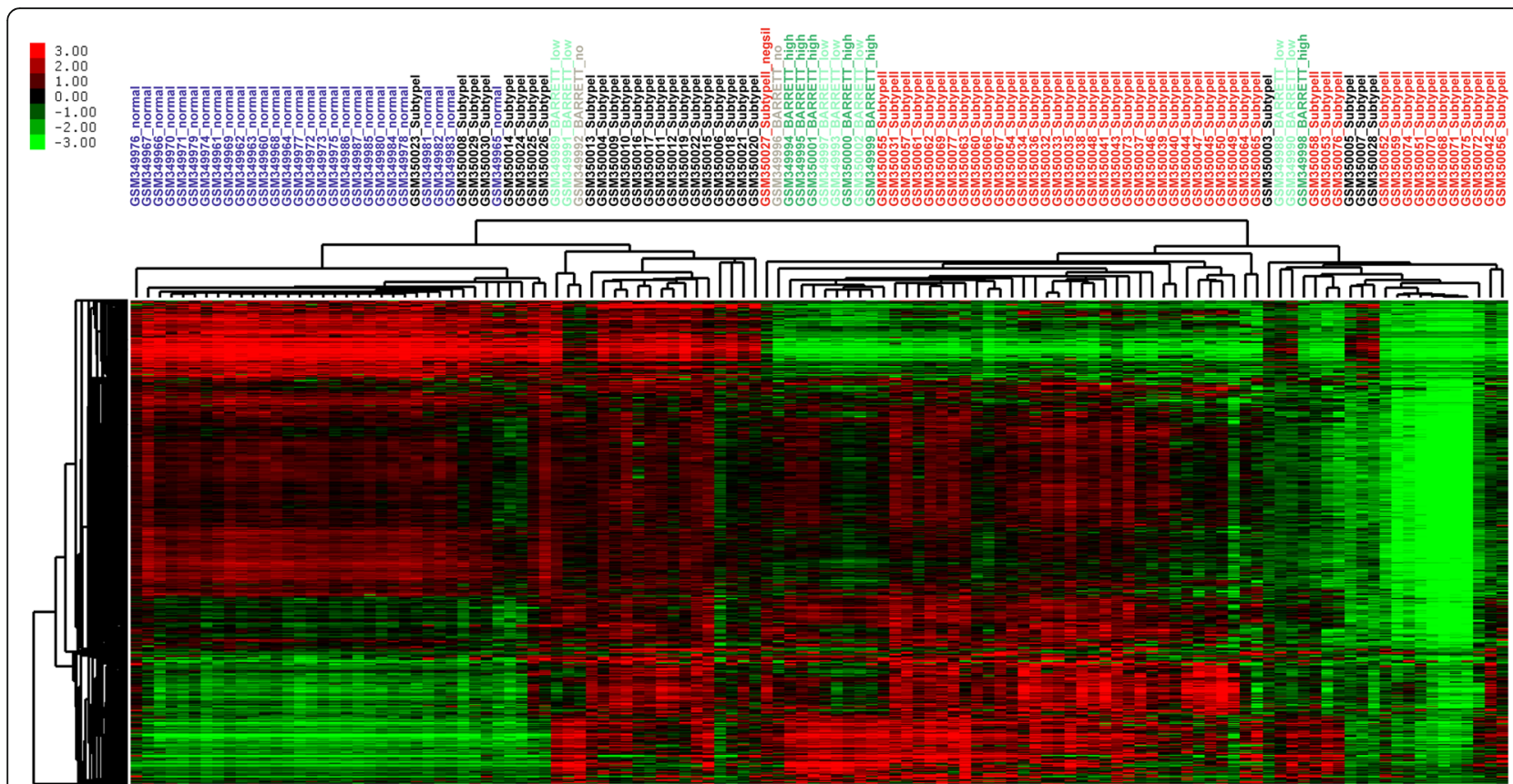

Fig. 3 Unsupervised hierarchical clustering of two subtypes of EAC, normal esophagus, and BE. "BARRETT_low" denotes low grade dysplasia in Barrett's esophagus, while "BARRETT_high" means high grade dysplasia in Barrett's esophagus. "BARRETT_no" stands for non-dysplastic Barrett's esophagus (data from GSE13898)

\section{Esophageal adenocarcinoma subtype-specific somatic mutations}

In the TCGA cohort, 87 of 88 esophageal adenocarcinoma profiled by RNA-Seq was also analyzed by exome-Seq, including 28 subtype I cases with positive silhouette values, 30 subtype II cases with positive silhouette values and 29 cases with negative silhouette values. An average of 254, 311 and 279 somatic mutations were identified in subtype I, subtype II cases with positive silhouette values and cases with negative silhouette values, respectively (Fig. 5). No significant difference in the numbers of mutation between subtype I and subtype II was found (Additional file 1: Table S6 and Additional file 2: Figure S1, $p=0.5522$, T-test).

When analyzed all these $87 \mathrm{EAC}$ cases as a population using MutSigCV analysis, 69 genes, including TP53, CDKN2A, MUC6, ARID1A, ERBB2 and SMAD4, were found significantly mutated in this cohort $(p<0.01)$. Four of these 69 genes (TP53, CDKN2A, ARID1A and $S M A D 4)$ were known somatic mutations in EAC.

To identify subtype specific somatic mutations, Mut$\mathrm{SigCv}$ analysis was performed on core subtype I and core subtype II cases, respectively. As showed, only TP53 and $C D K N 2 A$ genes were significantly mutated in both subtype I and subtype II EAC. Twenty-four genes, including SMAD4, SOCS4 and SKAP2, were only significantly mutated in subtype I EAC, while 30 genes, including $A R I D 1 A, D C D C 1$ and $I V L$, were only significantly somatically mutated in subtype II cases (Fig. 5). KEGG pathway analysis showed that genes significantly $\mathrm{mu}$ tated in subtype I EAC (TP53, CDKN2A and SMAD4) and genes significantly mutated in subtype II EAC (TP53, CDKN2A and $R B 1, C D C 7$ ) were significantly enriched in pathways including cell cycle.

\section{Association between molecular subtypes and response to chemotherapy}

Studying the association between molecular subtypes and response to therapies will help to guide the future treatment for EAC patients. In the TCGA dataset, excluding patients with negative silhouette values and thus less reliable subtype assignments, only three patients took chemotherapy. Among these three patients, two were from subtype II and both had a complete response to chemotherapy, while one was from subtype I and regrettably had clinical progressive disease, nevertheless the statistic test between subtypes and chemotherapy did not reach significance (Additional file 1: Table S1, $p=$ 0.0833). In addition, only one patient with positive silhouette values, who was from subtype II, took radiotherapy and had a complete response.

To further investigate the genes relevant to chemo-sensitivity/resistance, we compared the gene expression profiles between four EAC patients with complete responses to chemotherapy and three patients with progressive diseases from the TCGA data by SAMseq analysis (including the cases with negative silhouette values). As a result, 219 genes were significantly over-expressed in patients with 


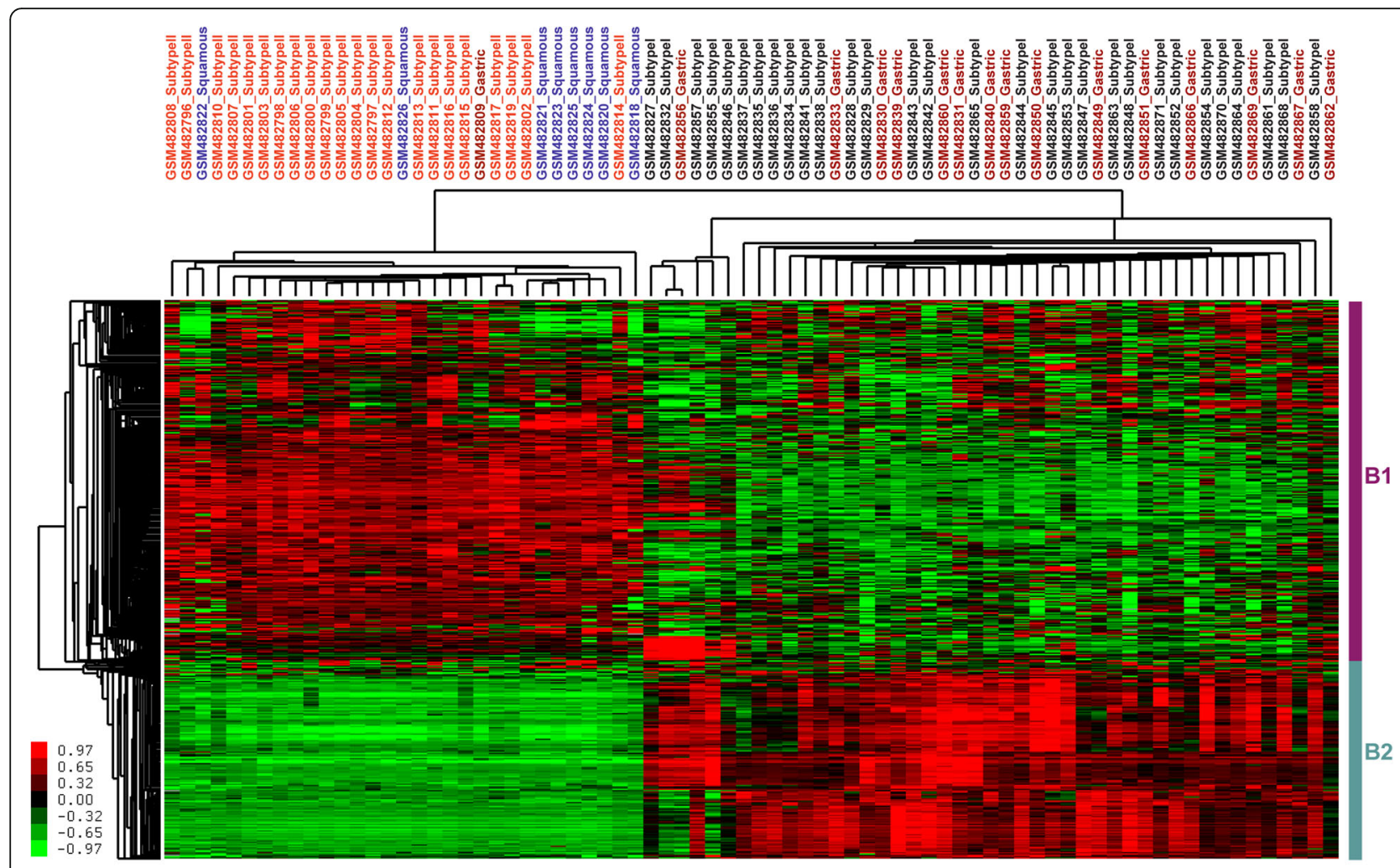

KEGG Pathway items enriched by genes from branch B1 and B2:

B1: Protein digestion and absorption

Drug metabolism - cytochrome P450

Metabolism of xenobiotics by cytochrome P450

Chemical carcinogenesis

ECM-receptor interaction

Cell adhesion molecules (CAMs)

Retinol metabolism

Focal adhesion

Steroid hormone biosynthesis

PI3K-Akt signaling pathway

etc. (Full list in supplementary Table s4)
B2: Measles

Drug metabolism - other enzymes

Retinol metabolism

Olfactory transduction

Chemical carcinogenesis

Herpes simplex infection

Fig. 4 Unsupervised hierarchical clustering of two subtypes of EAC, gastric and squamous carcinoma. "Gastric" labelled above heatmap denotes gastric adenocarcinoma while "Squamous" stands for squamous cell carcinoma (Data from GSE19417)

progressive diseases while no gene was over-expressed in patients with complete responses to chemotherapy. Forty-five of these 219 genes were also over-expressed in subtype I compared to subtype II EAC. These overlapped genes include ATP6VOA4, BMP7, KLK11, etc. (Additional file 1: Table S5), and were enriched in GO biological processes including proteolysis, epithelial cell differentiation and epithelium development.

\section{Identification of biomarkers for esophageal adenocarcinoma subtypes}

Identification of biomarkers for molecular subtypes of EAC will provide new insights to the future diagnosis of these subtypes and guide the subtype-specific and -effective therapies. GSEA and SAMseq analyses were used to identify potential markers for these two molecular subtypes. GSEA identified top 50 genes as potential biomarkers for each subtype while 678 genes were shown to be significantly over-expressed in subtype I than subtype II cases by SAMseq analysis. The comparison between GSEA and SAMseq found that all the top 50 genes from GSEA analysis for subtype I were also significantly over-expressed in subtype I than subtype II by SAMseq analysis (Fig. 6).

Among the top 50 genes, TP63 is a transcription factor from p53 family and was found to be significantly over-expressed in subtype I than subtype II EAC. The additional molecular test (from GSE13898) confirmed that all the subtype I EAC were TP63 positive while all the subtype II cases were TP63 negative, TP63 was very 


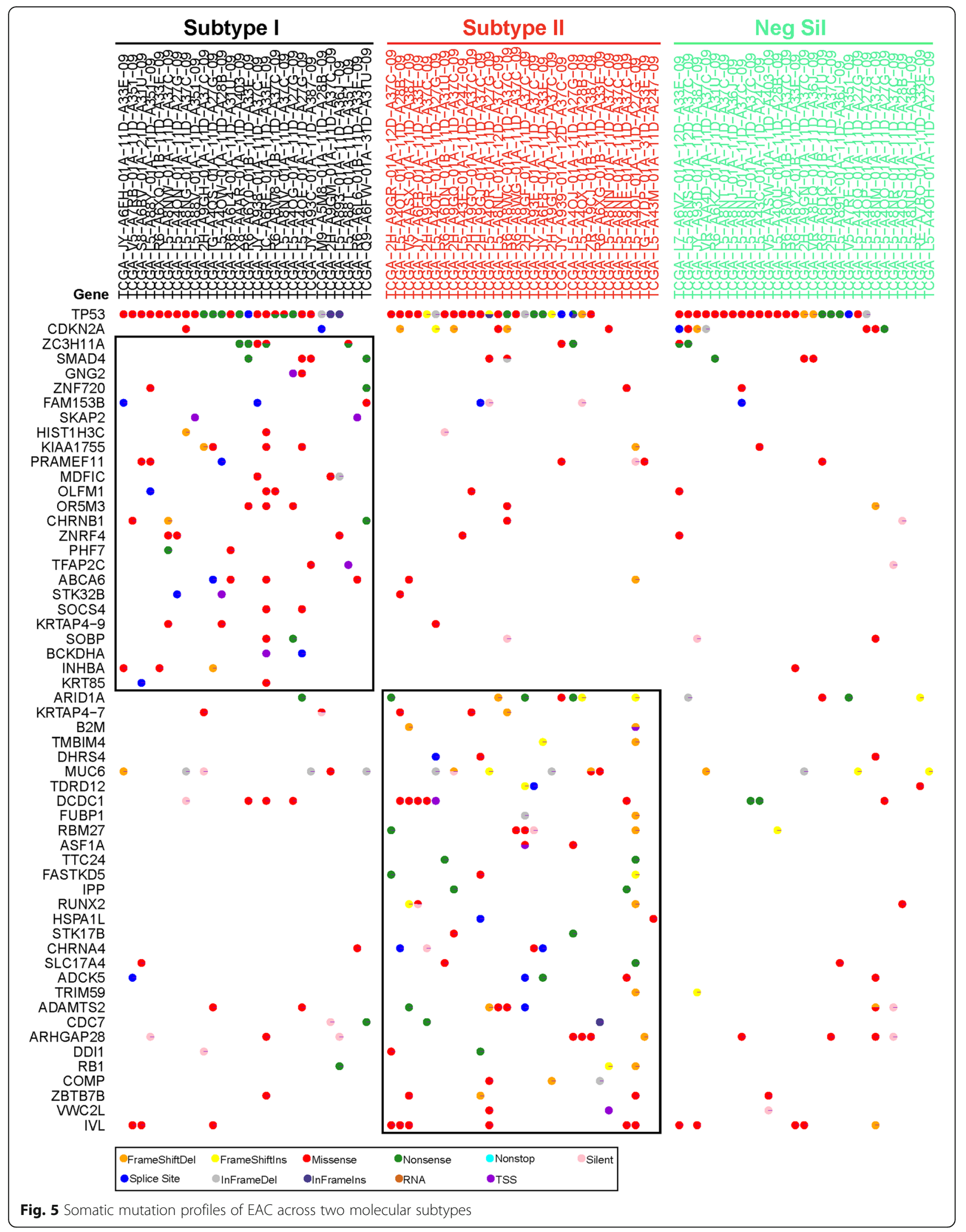




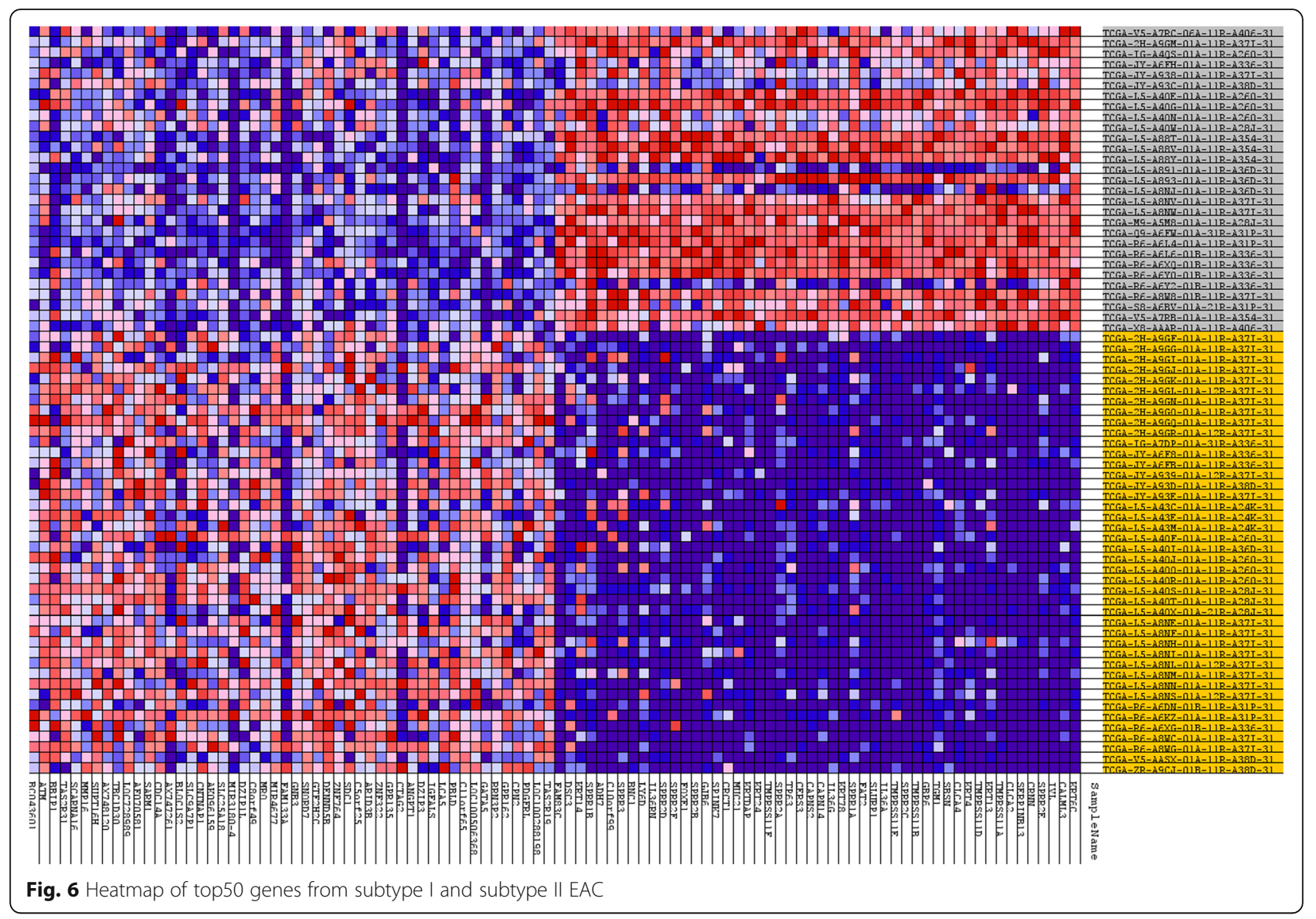

specific to subtype I EAC $(p<0.0001$, Additional file 1: Table S1) [21].

\section{Discussion}

Through meta-analysis, we identified and validated that there are two molecular distinct subtypes of EAC in three independent cohorts. Although these two subtypes had similar clinical-pathological characteristics including age, gender, grade, history of alcohol and smoking, degree of differentiation, they differed in expression profiles, mutation profiles and T-staging. Specifically, we identified TP63 was specific to subtype I EAC, suggesting a potential biomarker for further investigation and molecular analysis.

Gastrointestinal reflux has been reported to be one of the major causes for metaplasia of esophagus and BE [27]. The reflux activates the columnar differentiation pathway through gain of expression of intestinal transcription factors, including CDX1, CDX2, GATA4, GATA6, HNF1 $\alpha$, TGF $\beta$. Since these genes expressed in the similar levels between the two molecular subtypes, it suggested that the columnar differentiation might be activated for both molecular subtypes by gastrointestinal reflux. Meanwhile, the reflux would also inactivate the squamous differentiation pathway with expressional loss of some critical transcription factors, including TP63 and SOX2, which lead to the squamous de-differentiation manifested by the reduced expression of squamous differentiation markers, including keratin 4, keratin 14, and SPRRs. However, these squamous differentiation related genes were found to be significantly higher expressed in subtype I EAC than subtype II EAC cases, indicating that both molecular subtypes accompanied with the columnar differentiation but EAC from different molecular subtypes underwent different degrees of squamous de-differentiation. BE was reported to be the precursor of EAC. Interestingly, our results showed that subtype II EAC, not subtype I EAC, shared more common molecular expression patterns with $\mathrm{BE}$.

The landscape of somatic mutations in EAC has been widely analyzed [1, 28-31]. A number of genes were found to be significantly mutated in EAC in the earlier stages, including TP53, CDKN2A, SMAD4 and ARID1A [28]. By analyzing the somatic mutations in view of the two molecular subtypes based on the TCGA dataset, we found that TP53 and CDKN2A are as reported to be commonly mutated in the majority of EAC patients regardless of subtypes, indicating that TP53 mutations may be early events in the development of EAC $[1,29]$. 
Nevertheless, 24 and 30 genes were only significantly mutated in subtype I and subtype II EAC patients, respectively. SMAD4 and ARID1A have been reported to be significantly mutated in EAC patients previously [28]. However, within our current study, SMAD4 was only found to be significantly mutated in subtype I, in contrast, ARID1A was only significantly mutated in subtype II EAC patients, indicating EAC subtype-specific mutation profile and possible subtype-specific tumorigenesis mechanism. Although about two dozens of genes significantly mutated in each subtype, the cell cycle pathway was shown to be enriched in both mutated gene sets from subtype I (TP53, CDKN2A, SMAD4) and subtype II (TP53, CDKN2A, RB1, CDC7) EAC, indicating the important function of the cell-cycle pathway in both subtypes.

In this study, we also performed the primary analysis of chemotherapy response in EAC patients despite a limited number of patients have available therapeutic information. We showed that subtype II EAC was more sensitive to frontline chemotherapy while subtype I was more tolerant to the chemotherapy although the association between molecular subtypes and chemotherapy response did not reach significance, nevertheless it is still worth to measure the association between EAC molecular subtypes and chemotherapy in a larger sample size since the association assessment in current study was analyzed on only three patients. NGFR, known as nerve growth factor receptor, expressed in the basal cells of the normal esophageal epithelium and a subset of cells of ESCC, was found to be expressed higher in subtype I EAC than subtype II EAC. The NGFR+ cells in ESCC were more likely to be the cancer stem cell, and more resistant to chemotherapy drug DDP than NGFR- cells of ESCC [32]. This may also indicate the resistant roles of NGFR for the chemotherapy response in the treatment of subtype I EAC patients. In addition, NGFR was reported to be a potential biomarker for molecular subtypes of breast cancer, including basal-like breast cancer and luminal B breast cancer [33]. Future study targeting NGFR in EAC may not only help to stratify the molecular subtypes of EAC but may also help to overcome the chemotherapy resistance and develop novel targeted therapeutic methods for subtype I EAC patients. CXCR2 is a member of the G-protein-coupled receptor family and a receptor for IL8 (interleukin 8) and CXCL1. CXCL1 is a protein showing melanoma growth stimulating activity. In our study, CXCR2 was significantly over-expressed in subtype I EAC than subtype II EAC. A study targeting CXCR2 in EAC cell line demonstrated that inhibition of CXCR2 with a small molecular inhibitor (SB332235) suppressed the invasiveness of EAC derived OE33 cells [34], suggesting that subtype I EAC may exhibit high sensitivity to CXCR2 targeted therapy.
When comparing subtype I specific genes and chemoresistant relative genes, 45 genes, including ATP6VOA4, $B M P 7$ and $K L K 11$, were found to be over-expressed in both groups. ATP6VOA4 is one subunit of v-ATPase which was reported to be involved in chemo-resistance and invasion of tumor cells [35-37] and a biomarker for specific subtypes of human gliomas [35]. Also, ATP6 $V O A 4$ is one of the 45 genes highly expressed in subtype I and chemo-resistant EAC patients. ATP6V1C1, a family member of $A T P 6 V 0 A 4$, was highly expressed in $\mathrm{BE}$ and EAC, could be blocked by esomeprazole, resulted in antineoplastic effects and inhibition of proliferation, cell invasion and apoptosis of EAC cells [38]. In addition, ATP6V1C1 was also reported to display resistance to cytotoxic drugs [39-41], and therefore could be a potential therapeutic target for chemo-resistant tumor treatment [42-46]. BMP7, bone morphogenetic protein 7, was found to be consistently over-expressed in chemoresistant ovarian cell line than chemo-sensitive ovarian cell line [47]. By analyzing gene expression of clinical primary advanced colorectal cancer, Li et al. reported that expression signature of $H O X B 8$ and KLK11 could predict the effects of FOLFOX4 chemotherapy in primary advanced colorectal cancer patients [48]. The same research group also knocked down KLK11 in colorectal cancer cell line and shown that decreasing of KLK11 expression inhibited the cell proliferation and enhanced the sensitivity to oxaliplatin [49].

Our study suggested that subtype II EAC patients might be more likely responsive to chemo-therapy. However, because only limited clinical information, such as chemotherapy and TNM staging, from a small number of EAC patients available, this association between chemo-therapy and EAC molecular subtypes needs to be explained with caution. Given future analyses of more comprehensive clinical information from larger EAC patient populations support our findings, this subtype-specific chemo-therapy response would certainly help guide the appropriate clinical treatments for patients with different subtypes.

\section{Conclusions}

In summary, the analysis performed in this study identified and validated the two molecular subtypes of EAC with different expression and somatic mutation patterns. Meanwhile, the subtype II EAC was shown more sensitive to the frontline chemotherapy than subtype I EAC.

\section{Methods}

To identify and define the molecular subtypes of EAC, we collected the GEO datasets with plenty of EAC samples ( $\geq 50$ cases), including GSE13898 (75 EAC cases) [21] and GSE19417 (52 EAC cases) [22]. In addition, level 3 RNA-Seq and exome-Seq data of EAC (88 cases) were collected from TCGA database as well at March 
17, 2015 [50]. After filtering expression profile to genes by a standard deviation (SD), the Consensus Clustering ( $\mathrm{R}$ package ConsensusClusteringPlus) [51] for each of the three expression datasets was performed to determine the optimal number of subtypes and to assign the subtype for each EAC case, Consensus Clustering resampled samples/genes with ratio $80 \%$ and executed agglomerative hierarchical clustering, this process was ran over for 1,000 iterations as performed previously [3, $4,52]$. The statistical significance of subtypes in each dataset was examined by SigClust analysis [53]. Silhouette analysis ( $\mathrm{R}$ package cluster) [54] was then used to measure the accuracy of subtype assignment from ConsensusClusteringPlus. Subclass mapping was used to determine the reproducibility of molecular subtypes between three above expression profiling datasets [55]. Subtype specific gene expression patterns and pathways were analyzed by Gene Set Enrichment Analysis (GSEA) [56], DAVID Bioinformatics Resources [57, 58] and Significance Analysis of Microarrays (SAMseq) [59]. Cluster 3.0 was used to do hierarchical clustering [60] and clustering result was viewed by TreeView [61]. The significantly mutated genes were identified in entire EAC cohort or in subtype specific manner by MutSigCV analysis [62]. The contingency analyses performed in this study were assessed by the chi-square and Fisher exact tests using GraphPad Prism software and $p$ value less than 0.05 was considered significant.

\section{Additional files}

Additional file 1: Table S1. Clinical features of two EAC molecular subtypes. Table S2. Differentially expressed genes between two EAC molecular subtypes by SAMseq. Table S3. Biological processes annotation of Gene Ontology for differentially expressed genes from SAMseq. Table S4. KEGG Pathway annotation for differentially expressed genes from SAMseq. Table S5. Overlapped genes between subtype I specific genes and chemo-resistant genes. Table S6. The mean number of mutations per case in different EAC subtypes by TCGA exome-seq data. (XLSX 94 kb)

Additional file 2: Figure S1. The number of mutations per EAC case from different EAC subtypes. (TIFF $98 \mathrm{~kb}$ )

\section{Abbreviations}

BASAL set: HUPER_BREAST_BASAL_VS_LUMINAL_UP; BE: Barret's esophagus; EAC: Esophageal adenocarcinoma; EC: Esophageal carcinoma; ESCC: Esophageal squamous carcinoma; GSEA: Gene set enrichment analysis; WANG set:WANG_BARRETTS_ESOPHAGUS_AND_ESOPHAGUS_CANCER_DN

\section{Acknowledgements}

The results published or shown here are part based upon data generated by the TCGA Research Network: http://cancergenome.nih.gov/.

\section{Funding}

This work was supported by the National Natural Science Foundation of China (No. 81602362, to XG), the program for Science and Technology Development in Henan Province (No.162102310391, to XG), the program for Young Key Teacher of Henan Province (2016GGJS-214, to XG), the program for Innovative Talents of Science and Technology in Henan Province (No. 18HASTIT048, to XG), the supporting grants of Henan University
(No.2015YBZR048, No.yqpy20170039 and No.B2015151, to XG), and Yellow River Scholar Program (No.H2016012, to XG). The funding bodies were not involved in the study design, data collection, analysis and interpretation of data, or in writing of this manuscript.

\section{Availability of data and materials}

The datasets analyzed during the current study are available in the Gene Expression Omnibus (GEO) repository with accession ID GSE13898 and GSE19417, and TCGA database.

\section{Authors' contributions}

XG designed the study, analyzed the data, wrote the paper. YT and WZ analyzed the data, wrote the paper. All authors read and approved the final manuscript.

Ethics approval and consent to participate

Not applicable.

\section{Consent for publication}

Not applicable.

\section{Competing interests}

The authors declare that they have no competing interests.

\section{Publisher's Note}

Springer Nature remains neutral with regard to jurisdictional claims in published maps and institutional affiliations.

\section{Author details}

'Department of Preventive Medicine, Joint National Laboratory for Antibody Drug Engineering, Institute of Biomedical Informatics, School of Basic Medical Sciences, Henan University, Kaifeng 475004, China. ${ }^{2}$ Cell Signal Transduction Laboratory, Henan University, Kaifeng 475004, China. ${ }^{3}$ Department of Pathology, Stanford University School of Medicine, 300 Pasteur Drive, Stanford, CA 94305, USA. ${ }^{4}$ Department of Anesthesia, Stanford University, 300 Pasteur Drive, Stanford, CA 94305, USA.

Received: 30 May 2018 Accepted: 15 October 2018

Published online: 24 October 2018

\section{References}

1. Vaughan TL. From genomics to diagnostics of esophageal adenocarcinoma. Nat Genet. 2014:46:806-7.

2. Sinicrope FA, Shi Q, Smyrk TC, Thibodeau SN, Dienstmann R, Guinney J, et al. Molecular markers identify subtypes of stage III Colon Cancer associated with patient outcomes. Gastroenterology. 2015;148:88-99.

3. Guo X, Jo VY, Mills AM, Zhu SX, Lee CH, Espinosa I, et al. Clinically relevant molecular subtypes in Leiomyosarcoma. Clin Cancer Res. 2015;21:3501-11.

4. Guo X, Forgo E, van de Rijn M. Molecular subtyping of leiomyosarcoma with 3' end RNA sequencing. Genomics Data. 2015;5:366-7.

5. Damrauer JS, Hoadley KA, Chism DD, Fan C, Tiganelli CJ, Wobker SE, et al. Intrinsic subtypes of high-grade bladder cancer reflect the hallmarks of breast cancer biology. Proc Natl Acad Sci U S A. 2014;111:3110-5.

6. Choi W, Porten S, Kim S, Willis D, Plimack ER, Hoffman-Censits J, et al. Identification of distinct basal and luminal subtypes of muscle-invasive bladder cancer with different sensitivities to frontline chemotherapy. Cancer Cell. 2014;25:152-65.

7. Marisa L, de Reynies A, Duval A, Selves J, Gaub MP, Vescovo L, et al. Gene expression classification of colon cancer into molecular subtypes: characterization, validation, and prognostic value. PLoS Med. 2013;10: e1001453.

8. Lei Z, Tan IB, Das K, Deng N, Zouridis H, Pattison S, et al. Identification of molecular subtypes of gastric cancer with different responses to PI3-kinase inhibitors and 5-fluorouracil. Gastroenterology. 2013;145:554-65.

9. Perez-Villamil B, Romera-Lopez A, Hernandez-Prieto S, Lopez-Campos G, Calles A, Lopez-Asenjo JA, et al. Colon cancer molecular subtypes identified by expression profiling and associated to stroma, mucinous type and different clinical behavior. BMC Cancer. 2012;12:260.

10. The Cancer Genome Atlas Research Network. Integrated genomic analyses of ovarian carcinoma. Nature. 2011;474:609-15. 
11. Mills AM, Beck AH, Montgomery KD, Zhu SX, Espinosa I, Lee CH, et al. Expression of subtype-specific group 1 leiomyosarcoma markers in a wide variety of sarcomas by gene expression analysis and immunohistochemistry. Am J Surg Pathol. 2011;35:583-9.

12. Verhaak RG, Hoadley KA, Purdom E, Wang V, Qi Y, Wilkerson MD, et al. Integrated genomic analysis identifies clinically relevant subtypes of glioblastoma characterized by abnormalities in PDGFRA, IDH1, EGFR, and NF1. Cancer Cell. 2010;17:98-110.

13. Beck AH, Lee CH, Witten DM, Gleason BC, Edris B, Espinosa I, et al. Discovery of molecular subtypes in leiomyosarcoma through integrative molecular profiling. Oncogene. 2010;29:845-54.

14. Lapointe J, Li C, Higgins JP, van de Rijn M, Bair E, Montgomery K, et al. Gene expression profiling identifies clinically relevant subtypes of prostate cancer. Proc Natl Acad Sci U S A. 2004;101:811-6.

15. Sorlie T, Tibshirani R, Parker J, Hastie T, Marron JS, Nobel A, et al. Repeated observation of breast tumor subtypes in independent gene expression data sets. Proc Natl Acad Sci U S A. 2003:100:8418-23.

16. Shai R, Shi T, Kremen TJ, Horvath S, Liau LM, Cloughesy TF, et al. Gene expression profiling identifies molecular subtypes of gliomas. Oncogene. 2003;22:4918-23.

17. Sorlie T, Perou CM, Tibshirani R, Aas T, Geisler S, Johnsen H, et al. Gene expression patterns of breast carcinomas distinguish tumor subclasses with clinical implications. Proc Natl Acad Sci U S A. 2001;98:10869-74.

18. Perou CM, Sorlie T, Eisen MB, van de Rijn M, Jeffrey SS, Rees CA, et al. Molecular portraits of human breast tumours. Nature. 2000;406:747-52.

19. Cancer Genome Atlas N. Comprehensive molecular portraits of human breast tumours. Nature. 2012;490:61-70.

20. TCGA. http://cancergenome.nih.gov/.

21. Kim SM, Park YY, Park ES, Cho JY, Izzo JG, Zhang D, et al. Prognostic biomarkers for esophageal adenocarcinoma identified by analysis of tumor transcriptome. PLoS One. 2010;5:e15074.

22. Peters CJ, Rees JR, Hardwick RH, Hardwick JS, Vowler SL, Ong CA, et al. A 4gene signature predicts survival of patients with resected adenocarcinoma of the esophagus, junction, and gastric cardia. Gastroenterology. 2010;139: 1995-2004 e15.

23. Witt H, Mack SC, Ryzhova M, Bender S, Sill M, Isserlin R, et al. Delineation of two clinically and molecularly distinct subgroups of posterior fossa ependymoma. Cancer Cell. 2011;20:143-57.

24. Huper G, Marks JR. Isogenic normal basal and luminal mammary epithelial isolated by a novel method show a differential response to ionizing radiation. Cancer Res. 2007;67:2990-3001.

25. Wang S, Zhan M, Yin J, Abraham JM, Mori Y, Sato F, et al. Transcriptional profiling suggests that Barrett's metaplasia is an early intermediate stage in esophageal adenocarcinogenesis. Oncogene. 2006;25:3346-56.

26. Cancer Genome Atlas Research N, Analysis Working Group: Asan U, Agency BCC, Brigham, Women's H, Broad I, et al. Integrated genomic characterization of oesophageal carcinoma. Nature. 2017;541:169-75.

27. Chen H, Fang Y, Tevebaugh W, Orlando RC, Shaheen NJ, Chen X. Molecular mechanisms of Barrett's esophagus. Dig Dis Sci. 2011;56:3405-20.

28. Dulak AM, Stojanov P, Peng S, Lawrence MS, Fox C, Stewart C, et al. Exome and whole-genome sequencing of esophageal adenocarcinoma identifies recurrent driver events and mutational complexity. Nat Genet. 2013;45:478-86.

29. Weaver JM, Ross-Innes CS, Shannon N, Lynch AG, Forshew T, Barbera M, et al. Ordering of mutations in preinvasive disease stages of esophageal carcinogenesis. Nat Genet. 2014;46:837-43.

30. Agrawal N, Jiao Y, Bettegowda C, Hutfless SM, Wang Y, David S, et al. Comparative genomic analysis of esophageal adenocarcinoma and squamous cell carcinoma. Cancer Discovery. 2012;2:899-905.

31. Streppel MM, Lata S, DelaBastide M, Montgomery EA, Wang JS, Canto $\mathrm{Ml}$, et al. Next-generation sequencing of endoscopic biopsies identifies ARID1A as a tumor-suppressor gene in Barrett's esophagus. Oncogene. 2014;33:347-57.

32. Huang SD, Yuan Y, Liu XH, Gong DJ, Bai CG, Wang F, et al. Self-renewal and chemotherapy resistance of p75NTR positive cells in esophageal squamous cell carcinomas. BMC Cancer. 2009;9:9.

33. Tsang JY, Wong KH, Lai MW, Lacambra MD, Ko CW, Chan SK, et al. Nerve growth factor receptor (NGFR): a potential marker for specific molecular subtypes of breast cancer. J Clin Pathol. 2013;66:291-6.

34. Shrivastava MS, Hussain Z, Giricz O, Shenoy N, Polineni R, Maitra A, et al. Targeting chemokine pathways in esophageal adenocarcinoma. Cell Cycle. 2014;13:3320-7.
35. Gleize V, Boisselier B, Marie Y, Poea-Guyon S, Sanson M, Morel N. The renal $\checkmark$-ATPase a4 subunit is expressed in specific subtypes of human gliomas. Glia. 2012;60:1004-12.

36. Stock C, Schwab A. Protons make tumor cells move like clockwork. Pflugers Archiv. 2009;458:981-92.

37. Chiche J, Brahimi-Horn MC, Pouyssegur J. Tumour hypoxia induces a metabolic shift causing acidosis: a common feature in cancer. J Cell Mol Med. 2010;14:771-94.

38. Chueca E, Apostolova N, Esplugues JV, Garcia-Gonzalez MA, Lanas A, Piazuelo E. Proton pump inhibitors display antitumor effects in Barrett's adenocarcinoma cells. Front Pharmacol. 2016;7:452.

39. Murakami T, Shibuya I, Ise T, Chen ZS, Akiyama S, Nakagawa M, et al. Elevated expression of vacuolar proton pump genes and cellular $\mathrm{PH}$ in cisplatin resistance. Int J Cancer. 2001;93:869-74.

40. Luciani F, Spada M, De Milito A, Molinari A, Rivoltini L, Montinaro A, et al. Effect of proton pump inhibitor pretreatment on resistance of solid tumors to cytotoxic drugs. J Natl Cancer Inst. 2004;96:1702-13.

41. Marino ML, Fais S, Djavaheri-Mergny M, Villa A, Meschini S, Lozupone F, et al. Proton pump inhibition induces autophagy as a survival mechanism following oxidative stress in human melanoma cells. Cell Death Dis. 2010;1:e87.

42. Martinez-Zaguilan R, Raghunand N, Lynch RM, Bellamy W, Martinez GM, Rojas B, et al. pH and drug resistance. I. Functional expression of plasmalemmal V-type H+-ATPase in drug-resistant human breast carcinoma cell lines. Biochem Pharmacol. 1999;57:1037-46.

43. Sennoune SR, Bakunts K, Martinez GM, Chua-Tuan JL, Kebir Y, Attaya MN, et al. Vacuolar H+-ATPase in human breast cancer cells with distinct metastatic potential: distribution and functional activity. Am J Physiol Cell physiol. 2004:286:C1443-52.

44. Yeo M, Kim DK, Kim YB, Oh TY, Lee JE, Cho SW, et al. Selective induction of apoptosis with proton pump inhibitor in gastric cancer cells. Clin Cancer Res. 2004;10:8687-96.

45. Lu X, Qin W, Li J, Tan N, Pan D, Zhang H, et al. The growth and metastasis of human hepatocellular carcinoma xenografts are inhibited by small interfering RNA targeting to the subunit ATP6L of proton pump. Cancer Res. 2005;65:6843-9.

46. De Milito A, lessi E, Logozzi M, Lozupone F, Spada M, Marino ML, et al. Proton pump inhibitors induce apoptosis of human B-cell tumors through a caspase-independent mechanism involving reactive oxygen species. Cancer Res. 2007;67:5408-17.

47. Cheng L, Lu W, Kulkarni B, Pejovic T, Yan X, Chiang JH, et al. Analysis of chemotherapy response programs in ovarian cancers by the nextgeneration sequencing technologies. Gynecol Oncol. 2010;117:159-69.

48. Li S, Lu X, Chi P, Pan J. Identification of HOXB8 and KLK11 expression levels as potential biomarkers to predict the effects of FOLFOX4 chemotherapy. Future Oncol. 2013;9:727-36.

49. Xu Z, Chi P, Pan J, Shen S, Sun Y, Wang X, et al. Knockdown of KLK11 inhibits cell proliferation and increases oxaliplatin sensitivity in human colorectal cancer. Exp Ther Med. 2016;12:2855-60.

50. TCGA. https://portal.gdc.cancer.gov.

51. Wilkerson MD, Hayes DN. ConsensusClusterPlus: a class discovery tool with confidence assessments and item tracking. Bioinformatics. 2010;26:1572-3.

52. An $Y$, Wang $H$, Jie J, Tang $Y$, Zhang $W$, Ji S, et al. Identification of distinct molecular subtypes of uterine carcinosarcoma. Oncotarget. 2017;8(9):1587886. https://doi.org/10.18632/oncotarget.15032

53. Liu Y, Hayes D, Nobel A, Marron JS. Statistical Significance of Clustering for High-Dimension, Low-Sample Size Data. J Am Stat Assoc. 2012;103:1281-93.

54. Peter RJ. Silhouettes: a graphical aid to the interpretation and validation of cluster analysis. J Comput Appl Math. 1987;20:13.

55. Hoshida Y, Brunet JP, Tamayo P, Golub TR, Mesirov JP. Subclass mapping: identifying common subtypes in independent disease data sets. PLoS One. 2007;2:e1195.

56. Subramanian A, Tamayo P, Mootha VK, Mukherjee S, Ebert BL, Gillette MA, et al. Gene set enrichment analysis: a knowledge-based approach for interpreting genome-wide expression profiles. Proc Natl Acad Sci U S A. 2005;102:15545-50.

57. Huang DW, Sherman BT, Lempicki RA. Systematic and integrative analysis of large gene lists using DAVID bioinformatics resources. Nat Protoc. 2009;4:44-57.

58. Huang DW, Sherman BT, Lempicki RA. Bioinformatics enrichment tools: paths toward the comprehensive functional analysis of large gene lists. Nucleic Acids Res. 2009;37:1-13.

59. Li J, Tibshirani R. Finding consistent patterns: a nonparametric approach for identifying differential expression in RNA-Seq data. Stat Methods Med Res. 2011. 
60. de Hoon MJ, Imoto S, Nolan J, Miyano S. Open source clustering software. Bioinformatics. 2004;20:1453-4.

61. Saldanha AJ. Java Treeview--extensible visualization of microarray data. Bioinformatics. 2004:20:3246-8.

62. Lawrence MS, Stojanov P, Polak P, Kryukov GV, Cibulskis K, Sivachenko A, et al. Mutational heterogeneity in cancer and the search for new cancerassociated genes. Nature. 2013;499:214-8.

Ready to submit your research? Choose BMC and benefit from:

- fast, convenient online submission

- thorough peer review by experienced researchers in your field

- rapid publication on acceptance

- support for research data, including large and complex data types

- gold Open Access which fosters wider collaboration and increased citations

- maximum visibility for your research: over $100 \mathrm{M}$ website views per year

At $\mathrm{BMC}$, research is always in progress.

Learn more biomedcentral.com/submissions 